N. OKADA

KODAI MATH. J.

4 (1981), 82-96

\title{
A MARTINGALE PROBLEM ASSOCIATED WITH DIFFUSION OPERATORS IN A DOMAIN
}

\author{
BY NORIO OKADA
}

\section{$\S 1$. Introduction.}

For a given diffusion operator with continuous coefficients, we consider the solution $P_{s, x}$ of the martingale problem such that all paths starting from $x$ at time $s$ stay in a domain $\bar{D}$ in $[0, \infty) \times R^{d}$ for ever. That is, the support of $P_{s, x}$ is contained in $C([0, \infty), \vec{D})$. Under some regularity conditions, we shall obtain a necessary and sufficient condition for the existence of such a solution on $\bar{D}$. Results and precise formulations are stated in $\S 2$. In $\S 3$, the results of $\S 2$ and some lemmas for them will be proved. In $\S 4$, we shall consider some examples such that the uniqueness of solutions of $\S 2$ holds. In these examples sample paths stay on the boundary after hitting the boundary. To discuss processes with reflecting or entrance boundary, we need probably another approach.

I am grateful to Professors M. NAGASAwA and M. Motoo for their kind comments and advice.

\section{§2. Existence and related topics.}

Let $D$ be an open subset with the boundary $\partial D$ of $[0, \infty) \times R^{d}$ and assume that:

[A.2.1] There exists a $\phi(t, x) \in \mathscr{H}^{2}\left([0, \infty) \times R^{d}\right)$ such that $D=\{(t, x) \in[0, \infty)$ $\left.\times R^{d}: \phi(t, x)<0\right\}$ and $\partial D=\left\{(t, x) \in[0, \infty) \times R^{d}: \phi(t, x)=0\right\}$ with an additional condition $\left|\nabla_{x} \phi\right| \neq 0$ on $\partial D$, where $\mathscr{T}^{2}\left([0, \infty) \times R^{d}\right)=\left\{f(t, x): f \in C_{b}^{1}\left([0, \infty) \times R^{d}\right)\right.$ and $\partial f / \partial x_{\imath} \in C_{b}^{1}\left([0, \infty) \times R^{d}\right)$ for all $\left.\imath=1, \cdots, d\right\}$ and $\nabla_{x}=\left(\partial / \partial x_{1}, \cdots, \partial / \partial x_{d}\right)$.

From [A.2.1], we have that $D \cap\{s \leqq t<\infty\}$ is not empty for any $s \geqq 0$ and that $\phi(t, x)>0$ on $U \cap\left\{[0, \infty) \times R^{d}-\bar{D}\right\}$ for some neighborhood $U$ of $\partial D$.

Let $x(t, \omega)=\omega(t)$ for $\omega \in \Omega=C\left([0, \infty) \times R^{d}\right), M^{s}$ and $M_{t}^{s}$ be the $\sigma$-fields generated by $\{x(u): u \geqq s\}$ and $\{x(u): s \leqq u \leqq t\}$, respectively, and put the following assumption :

[A. 2. 2] $a(t, x)=\left(a_{\imath \jmath}(t, x)\right)_{\imath, \jmath=1, \ldots, d}:[0, \infty) \times R^{d} \rightarrow R^{d} \otimes R^{d}$ is bounded, continuous, symmetric and nonnegative definite; $b(t, x)=\left(b_{\imath}(t, x)\right)_{i=1, \ldots, d}:[0, \infty) \times R^{d}$ $\rightarrow R^{d}$ is bounded and continuous.

Recerved December 8, 1979 
We consider the following martingale problem $(\bar{D}, a, b,(s, x) ; s \leqq t)$ : Given $(s, x) \in \bar{D}$, find a probability measure $P_{s, x}$ on $\left(\Omega, M^{s}\right)$ such that $P_{s, x}[x(s)=x]=1$ and, for any $f \in C_{b}^{1,2}\left([0, \infty) \times R^{d}\right),\left(M_{f}^{s}(t), M_{t}^{s}, P_{s, x}: s \leqq t\right)$ is a martingale which satisfies

$$
P_{s, x}[(t, x(t)) \in \bar{D} \quad \text { for every } t \geqq s]=1,
$$

where

$$
M_{f}^{s}(t)=f(t, x(t))-\int_{s}^{t}\left(\frac{\partial f}{\partial u}+\frac{1}{2} \sum_{\imath, \jmath=1}^{d} a_{\imath \jmath} \frac{\partial^{2} f}{\partial x_{i} \partial x_{\jmath}}+\sum_{\imath=1}^{d} b_{i} \frac{\partial f}{\partial x_{\imath}}\right)(u, x(u)) d u .
$$

Here $\bar{D}$ denotes the closure of $D$ and $C_{b}^{1,2}\left([0, \infty) \times R^{d}\right)$ denotes the class of functions on $[0, \infty) \times R^{d}$ which together with their first $t$-derivative and first two $x$ derivatives are bounded and continuous.

For fixed $t$, let $D_{t}=\left\{x \in R^{d}:(t, x) \in D\right\}$ and $\partial D_{t}$ be the boundary of $D_{t}$ in $R^{d}$ and define

$$
\rho(t, x)=\left\{\begin{aligned}
\operatorname{dis}\left(x, \partial D_{t}\right) & \text { if } x \notin D_{t} \\
-\operatorname{dis}\left(x, \partial D_{t}\right) & \text { if } x \in D_{t},
\end{aligned}\right.
$$

where $\operatorname{dis}\left(x, \partial D_{t}\right)$ denotes the distance between $x$ and $\partial D_{t}$ in $R^{d}$. Then, from [A.2.1], $\rho(t, x)$ belongs to the class of $C_{b}^{1,2}$ in some neighborhood $U$ of $\partial D$ (see Lemma 3.1).

Now, we put the following two conditions:

$$
\begin{aligned}
& \sum_{\imath, j=1}^{d} a_{\imath j} \rho_{x_{\imath}} \rho_{x_{j}}(t, x)=0 \quad \text { on } \partial D ; \\
& H(t, x)=\frac{1}{2} \sum_{\imath, \jmath=1}^{d} a_{\imath j} \rho_{x_{i} x_{j}}(t, x)+\sum_{\imath=1}^{d} b_{i} \rho_{x_{\imath}}(t, x)+\rho_{t}(t, x) \\
& \leqq 0 \quad \text { on } \partial D,
\end{aligned}
$$

where $\rho_{x_{i}}=\partial \rho / \partial x_{\imath}, \rho_{x_{i} x}=\partial^{2} \rho / \partial x_{i} \partial x_{j}$ and $\rho_{t}=\partial \rho / \partial t$.

Our results are as follows.

Theorem 2.1. Assume [A.2.1] and [A.2.2]. Then the conditions [C.2.1] and [C.2.2] imply that there exists a solution $P_{s, x}$ of the martingale problem $(\bar{D}, a, b,(s, x) ; s \leqq t)$ satisfynng $(2.1)$ for every $(s, x) \in \bar{D}$. Conversely, the existence of the solution $P_{s, x}$ of the martingale problem $(\bar{D}, a, b,(s, x) ; s \leqq t)$ satisfying (2.1) for every $(s, x) \in \partial D$ implies the conditions [C.2.1] and [C.2.2].

Remark 2.1. It follows easily from Theorem 2.1 that, if the solution $P_{s, x}$ of the martingale problem $\left([0, \infty) \times R^{d}, a, b,(s, x) ; s \leqq t\right)$ is unique for all $(s, x)$ $\in[0, \infty) \times R^{d},(2.1)$ holds for each $(s, x) \in \bar{D}$.

If we replace the condition [C.2.2] by a stronger condition [C.2.3]: 


$$
H(t, x)=0 \quad \text { on } \partial D \text {, }
$$

then we have the following theorem.

Theorem 2.2. Assume [A.2.1] and [A.2.2]. Then the conditions [C.2.1] and [C.2.3] imply that there exists a solution $P_{s, x}$ of the martingale problem $(\partial D, a, b,(s, x) ; s \leqq t)$ satısfying

$$
P_{s, x}[(t, x(t)) \in \partial D \text { for every } t \geqq s]=1
$$

for every $(s, x) \in \partial D$. Conversely, the existence of the solution $P_{s, x}$ of the martingale problem $(\partial D, a, b,(s, x) ; s \leqq t)$ satısfying (2.2) for every $(s, x) \in \partial D$ implies the conditions [C.2.1] and [C.2.3].

Remark 2.2. The result of Theorem 2.2 contains those of Theorems 4.1 and 4.2 of Anderson [1] (see Remark 2.4).

Remark 2.3. It follows easily from Theorem 2.2 that, if the solution $P_{s, x}$ of the martingale problem $\left([0, \infty) \times R^{d}, a, b,(s, x) ; s \leqq t\right)$ is unique for every $(s, x)$ $\in[0, \infty) \times R^{d},(2.2)$ holds for each $(s, x) \in \partial D$.

Remark 2.4. By simple calculations, it follows that

$$
\begin{gathered}
\rho_{x_{\imath}}=\phi_{x_{i}}\left(\sum_{l=1}^{d} \phi_{x_{l}}^{2}\right)^{-1 / 2}, \\
\rho_{t}=\phi_{t}\left(\sum_{l=1}^{d} \phi_{x_{l}}^{2}\right)^{-1 / 2}
\end{gathered}
$$

and

$$
\begin{aligned}
\rho_{x_{i} x_{\jmath}}= & \left(\phi_{x_{i} x_{\jmath}} \sum_{l=1}^{d} \phi_{x_{l}}^{2}-\phi_{x_{\jmath}} \sum_{l=1}^{d} \phi_{x_{\imath} x_{l}} \phi_{x_{l}}-\phi_{x_{\imath}} \sum_{l=1}^{d} \phi_{x_{j} x_{l}} \phi_{x_{l}}\right. \\
& \left.+\phi_{x_{i}} \phi_{x_{\jmath_{l},}} \sum_{k=1}^{d} \phi_{x_{l} x_{k}} \phi_{x_{l}} \phi_{x_{k}}\right)\left(\sum_{l=1}^{d} \phi_{x_{l}}^{2}\right)^{-5 / 2}
\end{aligned}
$$

on $\partial D$, where $\phi_{t}=\partial \phi / \partial t, \phi_{x_{\imath}}=\partial \phi / \partial x_{\imath}$ and $\phi_{x_{i} x_{j}}=\partial^{2} \phi / \partial x_{\imath} \partial x_{\text {, }}$ (see Lemma 3.1). Combining those with [C.2.1] and the nonnegative definiteness of $a(t, x)$ on $\partial D$, we can claim that the conditions [C.2.2] and [C.2.3] are equivalent to the following conditions $[\mathrm{C} .2 .2]^{\prime}$ and $[\mathrm{C} .2 .3]^{\prime}$, respectively:

$$
\begin{aligned}
H^{\prime}(t, x)=\frac{1}{2} \sum_{\imath, \jmath=1}^{d} a_{\imath \jmath} \phi_{x_{i} x_{j}}(t, x) & +\sum_{i=1}^{d} b_{i} \phi_{x_{i}}(t, x) \\
& +\phi_{t}(t, x) \leqq 0 \text { on } \partial D ;
\end{aligned}
$$

$$
H^{\prime}(t, x)=0 \quad \text { on } \partial D .
$$

Remark 2.5. We may replace $\phi \in \mathscr{I}^{2}\left([0, \infty) \times R^{d}\right)$ by the one piecewise in 
$\mathscr{H}^{2}\left([0, \infty) \times R^{d}\right)$ with respect to $t \in[0, \infty)$. Indeed, if $\phi$ is not differentiable at some $T \in(s, \infty)$ with respect to $t$, we consider our problem on $[s, T]$ and $[T, \infty)$, respectively.

\section{$\S 3$. Proofs of results in $\S 2$.}

To prove the theorems, we prepare the following three lemmas.

LEMma 3.1. Suppose [A.2.1]. Denote the pornt $(t, y)$ such that dis $\left(x, \partial D_{t}\right)$ $=\operatorname{dis}(x, y), y \in \partial D_{t}$, by $\phi(t, x) \equiv\left(\psi_{i}(t, x)\right)_{i=0,1,2, \cdots, d}\left(x=\left(x_{1}, \cdots, x_{d}\right), y=\left(y_{1}, \cdots, y_{d}\right)\right.$ $\left.\in R^{d}\right)$. That is, $(t, y)=\phi(t, x)$. Then there is some nerghborhood $U$ of $\partial D$ such that $\phi_{i}(t, x), \imath=0,1,2, \cdots, d$, belongs to the class of $C_{b}^{1}$ on $U$. Moreover $\rho(t, x)$ belongs to the class of $C_{b}^{1,2}$ on $U$ and

$$
\begin{aligned}
& \rho_{t}(t, x)=\phi_{t}(\psi(t, x))\left\{\sum_{l=1}^{d} \phi_{x_{l}}^{2}(\psi(t, x))\right\}^{-1 / 2}, \\
& \rho_{x_{i}}(t, x)=\phi_{x_{i}}(\psi(t, x))\left\{\sum_{l=1}^{d} \phi_{x_{l}}^{2}(\psi(t, x))\right\}^{-1 / 2} \text { on } U .
\end{aligned}
$$

Proof. From [A.2.1], we can choose $\imath_{0} \in\{1, \cdots, d\}$ such that $\phi_{x_{\imath_{0}}} \neq 0$ for each $\left(t^{0}, x^{0}\right) \in \partial D$. Consider the following $d+1$ equations of $(t, x ; T, y), x=$ $\left(x_{1}, \cdots, x_{d}\right), y=\left(y_{1}, \cdots, y_{d}\right)$ :

$$
\left\{\begin{array}{l}
f_{i}(t, x ; T, y)=\left(y_{\imath_{0}}-x_{\imath_{0}}\right) \phi_{x_{i}}(T, y)-\left(y_{i}-x_{\imath}\right) \phi_{x_{\imath_{0}}}(T, y)=0 \text { for } 1 \leqq \imath \leqq \imath_{0}-1, \\
f_{\imath-1}(t, x ; T, y)=\left(y_{\imath_{0}}-x_{\imath_{0}}\right) \phi_{x_{i}}(T, y)-\left(y_{\imath}-x_{\imath}\right) \phi_{x_{\imath_{0}}}(T, y)=0 \text { for } \imath_{0}+1 \leqq \imath \leqq d, \\
f_{d}(t, x ; T, y)=\phi(T, y)=0 \\
f_{d+1}(t, x ; T, y)=T-t=0 .
\end{array}\right.
$$

The point $\left(t^{0}, x^{0} ; t^{0}, x^{0}\right)$ satisfies (3.3) and at the point the Jacobian

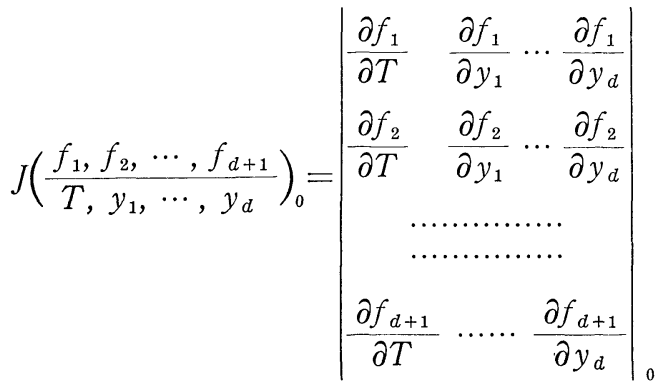

$$
\begin{aligned}
& =(-1)^{d+1+\imath_{0}} \phi_{x_{z_{0}}}^{d-2}\left(t^{0}, x^{0}\right)\left\{\sum_{l=1}^{d} \phi_{x_{l}}^{2}\left(t^{0}, x^{0}\right)\right\} \neq 0 \text {. }
\end{aligned}
$$

Then, by the implicit function theorem and $\partial D=\{\phi=0\}$, it is easily proved that 
we can choose some neighborhood $U\left(t^{0}, x^{0}\right)$ of $\left(t^{0}, x^{0}\right)$ such that $\psi(t, x)$ is uniquely determined and $(t, x ; \phi(t, x))$ satisfies (3.3) on $U\left(t^{0}, x^{0}\right)$. Furthermore, we have that the each component $\psi_{\imath}(t, x), \imath=0,1,2, \cdots, d$, of $\phi(t, x)$ belongs to the class of $C_{b}^{1}\left(U\left(t^{0}, x^{0}\right)\right)$ and

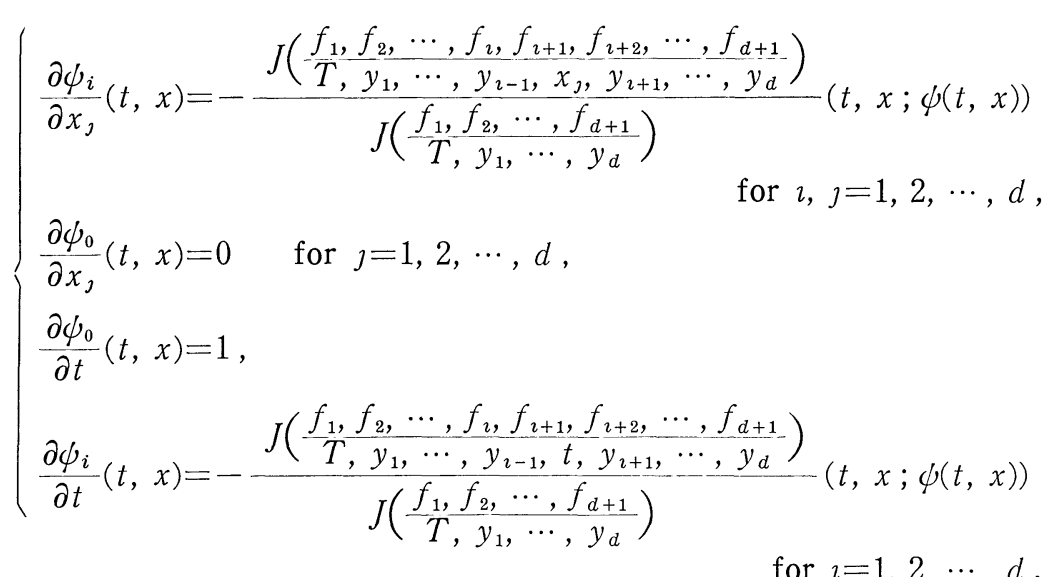

Next, using $\phi_{x_{i_{0}}} \neq 0$ on $U\left(t^{0}, x^{0}\right)$ and (3.3), it is obvious that, for each $(t, x)$ $\in U\left(t^{0}, x^{0}\right)$

$$
\rho(t, x)=\left\{\begin{aligned}
\operatorname{dis}(x, y) & \text { if }(t, x) \in U\left(t^{0}, x^{0}\right) \cap\left\{[0, \infty) \times R^{d}-\bar{D}\right\} \\
-\operatorname{dis}(x, y) & \text { if }(t, x) \in U\left(t^{0}, x^{0}\right) \cap \bar{D}
\end{aligned}\right.
$$

and

$$
\operatorname{dis}(x, y)=\left|\frac{\phi_{i_{0}}(t, x)-x_{i_{0}}}{\phi_{x_{i_{0}}}(\psi(t, x))}\right|\left\{\sum_{l=1}^{d} \phi_{x_{l}}^{2}(\psi(t, x))\right\}^{1 / 2}
$$

where $y=\left(\phi_{1}(t, x), \cdots, \phi_{d}(t, x)\right)$. But it follows from (3.4) and $\phi_{x_{i_{0}}}\left(t^{0}, x^{0}\right) \neq 0$ that

$$
\frac{\partial h}{\partial x_{\imath_{0}}}\left(t^{0}, x^{0}\right)=\frac{\sum_{l \neq \imath_{0}} \phi_{x_{l}}^{2}\left(t^{0}, x^{0}\right)}{\sum_{l=1}^{d} \phi_{x_{l}}^{2}\left(t^{0}, x^{0}\right)}-1<0
$$

where $h(t, x)=\phi_{\imath_{0}}(t, x)-x_{i_{0}}$. Hence, using the implicit function theorem and the fact that $\phi<0$ in $D, \phi>0$ in $[0, \infty) \times R^{d}-\bar{D}$ and $h=0$ on $\partial D$, we have that

$$
\rho(t, x)=\frac{\left(x_{\imath_{0}}-\phi_{i_{0}}(t, x)\right)}{\phi_{x_{\imath_{0}}}(\psi(t, x))}\left\{\sum_{l=1}^{d} \phi_{x_{l}}^{2}(\psi(t, x))\right\}^{1 / 2} \quad \text { for every }(t, x) \in U\left(t^{0}, x^{0}\right)
$$

and $\rho(t, x)$ belongs to the class of $C_{b}^{1}\left(U\left(t^{0}, x^{0}\right)\right)$. Furthermore, using (3.4) again, we have (3.1) and (3.2) on $U\left(t^{0}, x^{0}\right)$. From the form of (3.2), we conclude that $\rho(t, x)$ belongs to the class of $C_{b}^{1,2}\left(U\left(t^{0}, x^{0}\right)\right)$. At last, if we define $U=$ 
$\bigcup_{\left(t^{0}, x 0\right) \in \partial D} U\left(t^{0}, x^{0}\right)$, it is obvious that assertions of the lemma hold on $U$. Q. E. D.

Remark 3.1. We can consider the extended function of $\rho(t, x)$ such that it is equal to $\rho(t, x)$ of Lemma 3.1 on $U$, nonnegative on $\bar{D}$ and belongs to the class of $C_{b}^{1,2}\left([0, \infty) \times R^{d}\right)$. We denote the extended one by the same letter $\rho(t, x)$.

Lemma 3.2. Assume [A.2.1]. For each $(t, x) \in[0, \infty) \times R^{d}$, let $\tilde{\psi}(t, x)$ be the $(t, y)$ such that $\operatorname{dis}\left(x, \bar{D}_{t}\right)=\operatorname{dis}(x, y)$ and $y \in \bar{D}_{t}$. Then there is some nerghborhood $V$ of $\bar{D}$ such that $\tilde{\phi}(t, x)$ is continuous. Moreover $\tilde{\psi}$ is equal to $\psi$ on $V-D$ and equal to $(t, x)$ on $D$ (i. e. identical mapping on $\bar{D}$ ).

Proof. It is obvious that $V=U \cap D$ satisfies our assertions, where $U$ is of Lemma 3.1.

Q.E.D.

Lemma 3.3. Let $A$ be a closed subset of $R^{d+1}$. Let

$g(x)=\left(g_{\imath}(x)\right)_{i=1, \ldots, d}: R^{d+1} \rightarrow R^{d}$ be bounded, contrnuous and satısfynng $\sum_{i=1}^{d} g_{i}^{2}(x)$ $\neq 0$ on $A$;

$h(x): R^{d+1} \rightarrow R^{1}$ be bounded and contrnuous; and

$f(x)=\left(f_{i}(x)\right)_{i=1, \ldots, d}: A \rightarrow R^{d}$ be bounded, contınuous and satısfyıng

$$
\left(\sum_{i=1}^{d} f_{i} g_{i}+h\right)(x) \leqq 0(=0) \quad \text { on } A .
$$

Then there is a neighborhood $U$ of $A$ and a bounded vector function $F(x)=$ $\left(F_{i}(x)\right)_{\imath=1, . ., d}$, defined and contınuous on $R^{d+1}$, taking values in $R^{d}$ such that

$$
\left(\sum_{i=1}^{d} F_{i} g_{i}+h\right)(x) \leqq 0(=0) \quad \text { on } U
$$

and

$$
F=f \quad \text { on } A \text {. }
$$

Proof. For each $z \in A$, we can choose a number $i(z) \in\{1, \cdots, d\}$ and a neighborhood $U(z)$ of $z$ such that $g_{i(z)}(x) \neq 0$ for any $x \in \overline{U(z)}$. Let $\tilde{f}(x)=\left(\tilde{f}_{1}(x), \cdots\right.$, $\left.\tilde{f}_{d}(x)\right)$ be a continuous vector function defined on $R^{d+1}$, taking values in $R^{d}$ such that $\tilde{f}=f$ on $A$ and, for each $z \in A$, define a continuous vector function $\tilde{f}^{z}(x)=$ $\left(\tilde{f}_{1}^{z}(x), \cdots, \tilde{f}_{d}^{z}(x)\right)$ on $\overline{U(z)}$ such that

$$
\begin{aligned}
& \tilde{f}_{i}^{z}(x)=\tilde{f}_{i}(x) \quad \text { for } \imath \neq i(z), \\
& \tilde{f}_{i}^{z}(x)=\left(\frac{-h(x)-\sum_{\imath \neq(\tau)}^{d} \tilde{f}_{j}(x) g_{\jmath}(x)}{g_{i(z)}(x)}\right) \wedge \tilde{f}_{\imath(z)}(x) \\
& \text { for } \imath=i(z) \text { and } g_{i(z)}>0 \text { on } U(z),
\end{aligned}
$$




$$
\begin{aligned}
& \tilde{f}_{i}^{z}(x)=\left(\frac{-h(x)-\sum_{j \neq \imath(z)}^{d} \tilde{f}_{j}(x) g_{j}(x)}{g_{i(z)}(x)}\right) \vee \tilde{f}_{i(z)}(x) \\
& \text { for } \imath=i(z) \text { and } g_{i(z)}<0 \text { on } U(z) \\
& \left(\tilde{f}_{i}^{z}(x)=-\frac{h(x)+\sum_{j \neq i(z)}^{d} \tilde{\tilde{f}}_{j}(x) g_{j}(x)}{g_{i(z)}(x)} \text { for } \imath=i(z)\right) .
\end{aligned}
$$

Here $a \wedge b(a \vee b)$ denotes the minimum (maximum) of $a$ and $b$. Let $\left\{u^{z}(x) ; z \Subset A\right\}$ be a partition of unity over $A$, subordinate to the covering $\{U(z) ; z \in A\}$ of $A$. Then $F(x)=\sum_{z \in A} u^{z}(x) \tilde{f}^{z}(x)$ is defined and continuous on $R^{d+1}$ to $R^{d}$. Further, it is not difficult to show that the function $F(x)$ is equal to $f(x)$ on $A$ and $\sum_{i=1}^{d} F_{i}(x) g_{i}(x) \leqq-h(x)(=-h(x))$ on $U=\bigcup_{z \in A} U(z)$. Hence these $F(x)$ and $U$ are our seeking ones.

Q.E. D.

Proof of Theorem 2.1 [Existence part]. From Lemma 3.2, $a(\tilde{\psi}(t, x))$ is defined and continuous on some neighborhood $V$ of $\bar{D}$. Moreover, $a(\tilde{\psi}(t, x))$ (defined on $V$ ) can be extended to be a continuous, bounded, symmetric and nonnegative definite matrix on $[0, \infty) \times R^{d}$ and we denote this by the same letter $a(\tilde{\psi}(t, x))$. Then, by (3.2), Lemma 3.3 and [C.2.2], there is some neighborhood $W$ of $\partial D$ and a bounded, continuous $R^{d}$-vector function $\tilde{b}(t, x)=\left(\tilde{b}_{i}(t, x)\right)_{2=1, \ldots, d}$ on $[0, \infty) \times R^{d}$ such that

$$
\begin{aligned}
\tilde{H}(t, x)=\sum_{\imath=1}^{d} \tilde{b}_{i}(t, x) \rho_{x_{\imath}}(t, x)+\frac{1}{2} \sum_{\imath, j=1}^{d} a_{\imath j}(\tilde{\psi}(t, x)) \rho_{x_{\imath} x_{j}}(t, x) & \\
& +\rho_{t}(t, x) \leqq 0 \text { on } W-D
\end{aligned}
$$

and

$$
\tilde{b}(t, x)=b(t, x) \quad \text { on } \bar{D} \text {. }
$$

Since $a(\tilde{\psi})$ and $\tilde{b}$ are bounded and continuous on $[0, \infty) \times R^{d}$, there is a solution $P_{s, x}$ of the martingale problem $\left([0, \infty) \times R^{d}, a(\tilde{\psi}), \tilde{b},(s, x) ; s \leqq t\right)$ corresponding to $(a(\tilde{\psi}), \tilde{b})$ by the result of Strook and Varadhan [6]. We shall show that this $P_{s, x}$ satisfies (2.1). Let $f(z)$ be a nonnegative $C^{2}$-function on $R^{1}$ which is equal to one on $(-\infty, 0]$, smaller than one on $(0, \infty)$ and the first derivative of which is nonnegative on $R^{1}$. Further, let $\rho(t, x)$ be the one of Remark 3.1. Then,

$$
\begin{aligned}
& \frac{\partial f(\rho(t, x))}{\partial t}+\frac{1}{2} \sum_{i, j=1}^{d} a_{\imath j}(\tilde{\psi}(t, x)) \frac{\partial^{2} f(\rho(t, x))}{\partial x_{i} \partial x_{j}}+\sum_{i=1}^{d} \tilde{b}_{i}(t, x) \frac{\partial f(\rho(t, x))}{\partial x_{\imath}} \\
& =\frac{1}{2} f^{\prime \prime}(\rho(t, x)) \sum_{i, j=1}^{d} a_{\imath j}(\tilde{\psi}(t, x)) \rho_{x_{i}}(t, x) \rho_{x_{j}}(t, x)+f^{\prime}(\rho(t, x)) \\
& \cdot\left\{\frac{1}{2} \sum_{i, j=1}^{d} a_{\imath \jmath}(\tilde{\psi}(t, x)) \rho_{x_{i} x_{j}}(t, x)+\sum_{i=1}^{d} \tilde{b}_{x_{i}}(t, x) \rho_{x_{i}}(t, x)+\rho_{t}(t, x)\right\} .
\end{aligned}
$$


From (3.2), Lemma 3.2 and $\phi(\psi(t, x))=\psi(t, x) \in \partial D$ on $W$, we have

$$
\begin{aligned}
& \sum_{i, j=1}^{d} a_{\imath \jmath}(\tilde{\psi}(t, x)) \rho_{x_{i}}(t, x) \rho_{x_{j}}(t, x) \\
& \quad=\sum_{\imath, j=1}^{d} a_{\imath j}(\psi(t, x)) \cdot \rho_{x_{i}}(\psi(t, x)) \rho_{x_{j}}(\psi(t, x)) \quad \text { on } \bar{W}-\bar{D} .
\end{aligned}
$$

Hence, by [C.2.1], we have

$$
\sum_{\imath, \jmath=1}^{d} a_{\imath \jmath}(\tilde{\psi}(t, x)) \rho_{x_{\imath}}(t, x) \rho_{x_{j}}(t, x)=0 \quad \text { on } \bar{W}-\bar{D} .
$$

On the other hand, it follows easily that

$$
\begin{aligned}
f^{\prime}(\rho(t, x))\left\{\frac{1}{2} \sum_{\imath, \jmath=1}^{d} a_{\imath \jmath}(\tilde{\psi}(t, x)) \rho_{x_{i}}(t, x) \rho_{x_{j}}(t, x)\right. \\
\left.\quad+\sum_{i=1}^{d} \tilde{b}_{i}(t, x) \rho_{x_{i}}(t, x)+\rho_{t}(t, x)\right\} \\
=f^{\prime}(\rho(t, x)) \tilde{H}(t, x) \geqq 0 \quad \text { on } \bar{W}-\bar{D} .
\end{aligned}
$$

Further

$$
f^{\prime}(\rho(t, x))=f^{\prime \prime}(\rho(t, x))=0 \quad \text { on } \bar{D} .
$$

Hence it follows from (3.8)-(3.11) that

$$
\frac{\partial f(\rho)}{\partial t}+\frac{1}{2} \sum_{\imath, \jmath=1}^{d} a_{\imath}(\tilde{\psi}) \frac{\partial^{2} f(\rho)}{\partial x_{i} \partial x_{\jmath}}+\sum_{\imath=1}^{d} \tilde{b}_{i} \frac{\partial f(\rho)}{\partial x_{\imath}} \geqq 0 \quad \text { on } \bar{W} \cup \bar{D} \text {. }
$$

Let $\tau^{s}=\inf \{u \geqq s ;(u, x(u)) \notin W \cup D\}$. Then, by the martingale property and the optional sampling theorem, we have

$$
\begin{gathered}
E_{s, x}\left[f\left(\rho\left(t \wedge \tau^{s}, x\left(t \wedge \tau^{s}\right)\right)\right)\right]=f(\rho(s, x)) \\
+E_{s, x}\left[\int_{s}^{t \wedge \tau^{s}}\left\{\frac{\partial f(\rho)}{\partial u}+\frac{1}{2} \sum_{\imath, j=1}^{d} a_{\imath j}(\tilde{\psi}) \frac{\partial^{2} f(\rho)}{\partial x_{i} \partial x_{j}}+\sum_{\imath=1}^{d} \tilde{b}_{i} \frac{\partial f(\rho)}{\partial x_{\imath}}\right\}(u, x(u)) d u\right] \\
\geqq f(\rho(s, x))=1 \quad \text { for any }(s, x) \in \bar{D} \text { and any } t \geqq s .
\end{gathered}
$$

This implies that

$$
E_{s, x}\left[f\left(\rho\left(t \wedge \tau^{s}, x\left(t \wedge \tau^{s}\right)\right)\right)\right]=1 \quad \text { for any }(s, x) \in \bar{D} \text { and any } t \geqq s,
$$

because of $0 \leqq f(z) \leqq 1$. If $P_{s, x}$ has a positive measure on $B=\left\{\left(t \wedge \tau^{s}, x\left(t \wedge \tau^{s}\right)\right)\right.$ $\in W-\bar{D}\}$, it contradicts $(3.12)$ because of $f\left(\rho\left(t \wedge \tau^{s}, x\left(t \wedge \tau^{s}\right)\right)\right)<1$ on $B$. Hence we have

$$
P_{s, x}\left[\left(t \wedge \tau^{s}, x\left(t \wedge \tau^{s}\right)\right) \in \bar{D}\right]=1 \quad \text { for every } t \geqq s
$$

and immediately

$$
P_{s}, x_{x}[(t, x(t)) \in \bar{D} \text { for every } t \geqq s]=1 \quad \text { for each }(s, x) \in \bar{D},
$$


which is (2.1). Noting that $a(\tilde{\psi}(t, x))=a(t, x)$ and $\tilde{b}(t, x)=b(t, x)$ on $\bar{D}$, we have that this $P_{s, x}$ is the solution of our problem.

[Converse part] For each $(s, x) \in \partial D$, let $P_{s, x}$ be the solution of the martingale problem $(\bar{D}, a, b,(s, x) ; s \leqq t)$ satisfying (2.1).

At first, assume that [C.2.2] is not satisfied. That is, there is a point $(s, x)$ $\in \partial D$ such that $H(s, x)>0$. Then, by the continuity of $H(t, y)$, there are positive constants $\varepsilon$ and $\delta$ such that $H(t, y) \geqq \delta$ on the $\varepsilon$-neighborhood $U_{\varepsilon}$ of $(s, x)$. Let $\tilde{\tau}^{s}=\inf \left\{u \geqq s:(u, x(u)) \notin U_{\varepsilon}\right\}$. Then, by the optional sampling theorem,

$$
0 \leqq E_{s, x}\left[-\rho\left(t \wedge \tilde{\tau}^{s}, x\left(t \wedge \tilde{\tau}^{s}\right)\right)\right]=E_{s, x}\left[\int_{s}^{t \wedge \tilde{\tau}^{s}}-H(u, x(u)) d u\right]
$$

Hence it follows that

$$
\leqq-\delta E_{s, x}\left[t \wedge \tilde{\tau}^{s}-s\right] \quad \text { for any } t \geqq s \text {. }
$$

$$
E_{s, x}\left[t \wedge \tilde{\tau}^{s}-s\right]=0 \quad \text { for every } t \geqq s .
$$

Let $t>s$. Then, from (3.13), we have $\tilde{\tau}^{s}=s$ a.s in $P_{s, x}$ and it contradicts the continuity of the path functions. Hence we get [C.2.2] on $\partial D$.

Next, assume that [C.2.1] is not fulfilled. That is, there is a point $(s, x) \in \partial D$ such that

$$
\sum_{\imath, j=1}^{d} a_{\imath j} \rho_{x_{i}} \rho_{x_{j}}(s, x)>0 .
$$

Define, for a positive constant $\alpha, \hat{b}(t, y)=\left(\hat{b}_{i}(t, y)\right)_{i=1, \ldots, d}$ such that

$$
\hat{b}_{\imath}(t, y)=b_{\imath}(t, y)+\alpha \sum_{j=1}^{d} a_{\imath j} \rho_{x_{j}}(t, y) .
$$

Then, applying the transformation of the drift to the $P_{s, x}$, we have a solution $\hat{P}_{s, x}$ of the martingale problem corresponding to $(a, \hat{b})$ and satisfying (2.1) for each $(s, x) \in \partial D$. For sufficiently large $\alpha$, we have by (3.14) that

$$
\begin{aligned}
& \hat{H}(s, x)=\left\{\frac{1}{2} \sum_{\imath, j=1}^{d} a_{\imath j} \rho_{x_{i} x_{j}}+\sum_{\imath=1}^{d} \hat{b}_{\imath} \rho_{x_{\imath}}+\rho_{t}\right\}(s, x) \\
& =\left\{\frac{1}{2} \sum_{\imath, j=1}^{d} a_{\imath j} \rho_{x_{i} x_{j}}+\sum_{i=1}^{d} b_{i} \rho_{x_{\imath}}+\rho_{t}\right\}(s, x)+\alpha \sum_{\imath, j=1}^{d} a_{\imath j} \rho_{x_{i}} \rho_{x_{j}}(s, x)>0 .
\end{aligned}
$$

From previous result, (3.15) denies that this $\hat{P}_{s, x}$ satisfies (2.1). Hence [C.2.1] has to hold on $\partial D$ and the proof is finished.

Q.E. D.

Proof of Theorem 2.2 [Existence part]. From Lemma 3.1, $a(\psi(t, x))$ is defined on some neighborhood $V$ of $\partial D$. Then, by (3.2), Lemma 3.3 and [C.2.3], there are a neighborhood $U$ (contained in $V$ ) of $\partial D$ and a bounded and continuous $R^{d}$-vector function $\tilde{b}(t, x)=\left(\tilde{b}_{i}(t, x)\right)_{i=1, \ldots, d}$ on $[0, \infty) \times R^{d}$ such that

$$
\sum_{i=1}^{d} \tilde{b}_{i}(t, x) \rho_{x_{i}}(t, x)+\rho_{t}(t, x)+\frac{1}{2} \sum_{\imath, j=1}^{d} a_{\imath j}(\psi(t, x)) \rho_{x_{i} x_{j}}=0 \quad \text { on } U
$$


and

$$
\tilde{b}(t, x)=b(t, x) \quad \text { on } \partial D \text {. }
$$

Extend $a(\phi(t, x))$ from $U$ to $[0, \infty) \times R^{d}$ so that there exists a solution $P_{s, x}$ of the martingale problem corresponding to the extended $a(\psi(t, x))$ and $\tilde{b}(t, x)$ for each $(s, x) \in \partial D$. Let $\hat{\tau}^{s}=\inf \{u \geqq s:(u, x(u)) \notin U\}$ and $v(z)$ be a nonnegative $C^{2}$-function on $R^{1}$ which is equal to one at $\{0\}$ and smaller than one elsewhere. Then, using $v(\rho(t, x)), \hat{\tau}^{s}$ and (3.16) and arguing along the lines of the proof of Theorem 2.1, we have

$$
P_{s, x}[(t, x(t)) \in \partial D \text { for every } t \geqq s]=1 .
$$

Consequently, $P_{s, x}$ is the desired solution.

[Converse part] The next two facts are obvious. That is, both of the boundaries of the open domains $D$ and $[0, \infty) \times R^{d}-\bar{D}$ are $\partial D$; and solutions which satisfy (2.2) also satisfy (2.1) and the next equality:

$$
P_{s, x}\left[(t, x(t)) \in[0, \infty) \times R^{d}-D \text { for every } t \geqq s\right]=1 .
$$

Hence, applying the converse part of Theorem 2.1 to domains $D$ and $[0, \infty) \times$ $R^{d}-\bar{D}$ respectively, we have the conditions [C.2.1] and [C.2.3] on $\partial D$. Q. E. D.

Remark 3.2. In the above theorems, the assumption [A.2.2] guarantees the existence of the solution corresponding to the extended coefficients. The boundedness condition of the coefficients $a(t, x)$ and $b(t, x)$ in assumption may be replaced by the following weaker condition: for each $T>0$, there is some positive constant $L_{T}$ such that

$$
\begin{aligned}
& \sum_{\imath, j=1}^{d}\left|a_{\imath j}(t, x)\right|^{2}+\sum_{i=1}^{d}\left|b_{i}(t, x)\right|^{2} \leqq L_{T}\left(1+|x|^{2}\right) \\
& \qquad \text { for all }(t, x) \in[0, \infty) \times R^{d} \text { (refer to [5]). }
\end{aligned}
$$

Remark 3.3. Under [A.2.1], the local existence of the solution $P_{s, x}$ satisfying (2.1) (or (2.2)) is obtained more easily than the global existence of the solution satisfying (2.1) $((2.2))$ which we get in the above theorems. Moreover, by a general selection theorem, we can choose a family of solutions $\left\{P_{s, x} ;(s, x) \in\right.$ $\left.[0, \infty) \times R^{d}\right\}$ which is measurable with respect to $(s, x)$ for any $B \in M^{s}$. Hence, by Theorem A1.6 in [1], we get the solution $P_{s, x}$ of the martingale problem satisfying $(2.1)((2.2))$ for each $(s, x) \in \bar{D}(\partial D)$ under [A.2.2], which is another proof of the existence part of above theorems. But, if we consider our problem in terms of stochastic differential equation with continuous coefficients for an arbitrary initial distribution (including the explosion time and, furthermore, with boundary conditions), our approach in the above theorems is useful. 


\section{$\S 4$. Some results for the uniqueness problem.}

In this section, we consider the uniqueness problem of the solution of the martingale problem on $\bar{D}$. But, in practice, we treat only the case where the general condition for the uniqueness fails on the boundary $\partial D$ and the path stays on $\partial D$ after it hits $\partial D$.

Let $D$ be an open subset of $[0, \infty) \times R^{d}$ with the boundary $\partial D$ and put the following assumptions.

[A.4.1] Assume that, for each $(s, x) \in D$, the solution of the martingale problem $(\bar{D}, a, b,(s, x) ; s \leqq t)$ satisfying $(2.1)$ is uniquely determined on $M_{\sigma s \wedge t}^{s}$ for any $t \geqq s$, where $a(t, x)$ is bounded, measurable, symmetric and nonnegative definite on $[0, \infty) \times R^{d}, b(t, x)$ bounded and measurable on $[0, \infty) \times R^{d}, \sigma^{s}=$ $\inf \{u \geqq s:(u, x(u)) \in \partial D\}$ and $M_{\sigma s \wedge t}^{s}$ is a $\sigma$-field associated with $\sigma^{s} \wedge t$.

[A.4.2] Assume that, for each $(s, x) \in \partial D$, the solution of the martingale problem $(\partial D, a, b,(s, x) ; s \leqq t)$ satisfying (2.2) is unique.

Remark 4.1. As is well known, it is a sufficient condition for [A.4.1] that $b(t, x)$ and a matrix $\sigma(t, x)$ such that $a(t, x)=\sigma(t, x) \sigma(t, x)^{*}$ are local Lipschitz continuous with respect to the space variables $x$ on $D$ or that $a(t, x)$ is continuous and strictly elliptic on $D$. Here the local Lipschitz continuity means the Lipschitz continuity on every compact set contained in $D$ and $\sigma(t, x)^{*}$ denotes the transpose of $\sigma(t, x)$. On the other hand, it is a sufficient condition for [A.4.2] that $b(t, x)$ and a matrix $\sigma(t, x)$ such that $a(t, x)=\sigma(t, x) \sigma(t, x)^{*}$ are Lipschitz continuous on $\partial D$ with respect to the space variables $x$ or that $a(t, x)$ is continuous on $\partial D$ and, for all $(t, x) \in \partial D$ and $\theta \in R^{d}$ subject to $\left\langle\nabla_{x} \phi(t, x), \theta\right\rangle=0$, there is a positive constant $C_{1}$ independent of $(t, x)$ and $\theta$ such that $\langle a \theta, \theta\rangle(t, x) \geqq C_{1}|\theta|^{2}$. (For the detailed proofs, refer, e.g., to Theorem 1 in [3] and Theorem 3.2 in [1].)

Then we have the following theorem.

THEOREM 4.1. Assume [A.2.1], [A.4.1] and [A.4.2] and, furthermore, that the condition [C.4.1] holds:

[C.4.1] There are a neighborhood $U$ of $\partial D$, a positvve constant $C_{2}$ and $a$ bounded vector function $d(t, x) \in R^{d}$ on $\bar{D}$ such that

$$
\frac{1}{2} \sum_{i, j=1}^{d} a_{\imath j} \rho_{x_{i} x_{j}}+\sum_{i=1}^{d} \bar{b}_{i} \rho_{x_{i}}+\rho_{t} \geqq C_{2} \rho \quad \text { on } U \cap \bar{D},
$$

where $\bar{b}(t, x)=b(t, x)-a d(t, x)$.

Then there is at most one solution $P_{s, x}$ of the martingale problem $(\bar{D}, a, b,(s, x)$; $s \leqq t)$ satisfying (2.1) for each $(s, x) \in \bar{D}$. 
Proof. It follows from [A.4.1] and [A.4.2] that, if we see the fact that the path starting from the boundary $\partial D$ and staying in $\bar{D}$ never enters the interior $D$, we get the uniqueness of the solution of the martingale problem $(\bar{D}, a, b,(s, x)$; $s \leqq t)$ in the sense of (2.1) by the same way as that in Theorem 1 of [3]. Then we shall show that [C.4.1] guarantees this fact. At first, assume $d(t, x) \equiv 0$. Let $\tilde{\sigma}^{s}=\inf \{u \geqq s:(u, x(u)) \in \partial U \cap D\}$ and, for each $(s, x) \in \partial D, P_{s, x}$ be the solution of the martingale problem $(\bar{D}, a, b,(s, x) ; s \leqq t)$ satisfying (2.1). From (4.1) and the martingale property, we have

$$
\begin{aligned}
& E_{s, x}\left[-\rho\left(t \wedge \tilde{\sigma}^{s}, x\left(t \wedge \tilde{\sigma}^{s}\right)\right)\right] \\
& =E_{s, x}\left[\int_{s}^{t \wedge \tilde{\sigma}^{s}}\left\{-\sum_{\imath, \jmath=1}^{d} a_{\imath \jmath} \rho_{x_{i} x_{\jmath}}-\sum_{\imath=1}^{d} b_{i} \rho_{x_{\imath}}-\rho_{t}\right\}(u, x(u)) d u\right] \\
& \leqq C_{2} \int_{s}^{t} E_{s, x}\left[-\rho\left(u \wedge \tilde{\sigma}^{s}, x\left(u \wedge \tilde{\sigma}^{s}\right)\right)\right] d u \quad \text { for any } t \geqq s .
\end{aligned}
$$

This implies that $E_{s, x}\left[-\rho\left(t \wedge \tilde{\sigma}^{s}, x\left(t \wedge \tilde{\sigma}^{s}\right)\right)\right]=0$ and, consequently, $P_{s, x}[(t, x(t)) \in$ $\partial D$ for every $t \geqq s]=1$ for each $(s, x) \in \partial D$. Next, by the transformation of the drift, the case of the general $d(t, x)$ is reduced to the case $d(t, x) \equiv 0$. Hence the proof is completed.

Q.E. D.

Remark 4.2. We can easily see that it is a sufficient condition (but not a necessary one) for [C.4.1] that $a(t, x)$ and $b(t, x)$ are Lipschitz continuous with respect to the space variables $x$ on $U \cap \bar{D}$ for a neighborhood $U$ of $\partial D, \phi$ belongs to $\mathscr{T}^{3}\left([0, \infty) \times R^{d}\right)$ and [C.2.1] and [C.2.2] hold on $\partial D$. Here $\mathscr{T}^{3}\left([0, \infty) \times R^{d}\right)=$ $\left\{f(t, x): f \in C_{b}^{1}\left([0, \infty) \times R^{d}\right)\right.$ and $\partial f / \partial x_{i} \in C_{b}^{1,2}\left([0, \infty) \times R^{d}\right)$ for all $\left.\imath=1, \cdots, d\right\}$.

Combining Theorem 2.1 and Theorem 4.1, we get the following corollary immediately.

Corollary 4.1. Suppose that [A.2.1], [A.2.2], [A.4.1], [A.4.2], [C.2.1], [C.2.2] and [C.4.1] hold. Then there uniquely exists the solution $P_{s, x}$ of the martingale problem $(\bar{D}, a, b,(s, x)$; $s \leqq t)$ satısfying (2.1) for each $(s, x) \in \bar{D}$.

Remark 4.3. In Corollary 4.1, we have [C.2.3] inevitably.

Remark 4.4. From Corollary 4.1, we get the diffusion process $\left(\tilde{\Omega}, \tilde{M}^{0}, \tilde{M}^{s}\right.$, $\left.\tilde{M}_{t}^{s}, P_{s, x} ;(s, x) \in \bar{D}\right)$ on $\bar{D}$ which has the Feller property on $\bar{D}$ (cf. [6]), where $\tilde{\Omega}=\{\omega:(t, x(t)) \in \bar{D}$ for every $t \geqq 0\}, \tilde{M}^{s}=M^{s}[\cap \widetilde{\Omega}]$ and $\tilde{M}_{t}^{s}=M_{t}^{s}[\cap \tilde{\Omega}]$.

At last, we state a result of the martingale problem on all space $[0, \infty) \times R^{d}$ which is similar to that of Corollary 4.1. But there is an essential difference between the proofs of them.

Instead of [A.4.1], we put the following assumption:

[A.4.1 $]^{\prime}$ Assume that the solution of the martingale problem $\left([0, \infty) \times R^{d}\right.$, 
$a, b,(s, x) ; s \leqq t)$ is uniquely determined on $M_{\sigma s \wedge t}^{s}$ for every $t \geqq s$.

Proposition 4.1. Let $D$ be an open subset of $[0, \infty) \times R^{d}$ which satısfies [A.2.1] with the further condition $\phi \in \mathscr{H}^{3}\left([0, \infty) \times R^{d}\right)$ and assume [A.2.2], [A.4.1]', [A.4.2], [C.2.1], [C.2.2], [C.4.1] for $\bar{D}$ and the following condition:

[C.4.2] There is some nerghborhood $W$ of $\partial D$ such that $a(t, x)$ and $b(t, x)$ are Lipschitz contınuous with respect to the space variable $x$ on $\overline{W-D}$.

Then there uniquely exists the solution $P_{s, x}$ of the martingale problem $([0, \infty)$ $\left.\times R^{d}, a, b,(s, x) ; s \leqq t\right)$ for each $(s, x) \in[0, \infty) \times R^{d}$.

Proof. It is sufficient to prove that the path starting from the boundary $\partial D$ never leaves $\partial D$. From [C.2.1], [C.2.2]; [C.4.2] and $\phi \in \mathscr{H}^{3}\left([0, \infty) \times R^{d}\right)$, we have easily that

$$
\sum_{\imath, \jmath=1}^{d} a_{\imath \jmath} \rho_{x_{\imath}} \rho_{x_{j}}(t, x) \leqq C_{3} \rho(t, x)
$$

and

$$
H(t, x)=\frac{1}{2} \sum_{\imath, j=1}^{d} a_{\imath j} \rho_{x_{i}} \rho_{x_{j}}(t, x)+\sum_{i=1}^{d} b_{\imath} \rho_{x_{i}}(t, x)+\rho_{t}(t, x) \leqq C_{4} \rho(t, x)
$$

for some positive constants $C_{3}$ and $C_{4}$ on $W-D$. Let $P_{s, x}$ be the solution of the martingale problem $\left([0, \infty) \times R^{d}, a, b,(s, x) ; s \leqq t\right)$ and $\tau^{s}=\inf \{u \geqq s:(u, x(u)) \notin$ $W \cup D\}$. Choose nonnegative $C^{2}$ functions $\lambda_{n}(\xi)$ on $R^{1}$ such that $\lambda_{n}(\xi)$ increases to $0 \vee \xi$ as $n \rightarrow \infty, \lambda_{n}(\xi)=0$ in a neighborhood of $(-\infty, 0], 0 \leqq \lambda_{n}^{\prime}(\xi) \leqq 1$ on $R^{1}$ and $0 \leqq \lambda_{n}^{\prime \prime}(\xi) \leqq 2 n^{-1} \xi^{-1}$ on $(0, \infty)$ (see [7]). Then we have, for each $(s, x) \in \partial D$,

$$
\begin{aligned}
& E_{s, x}\left[\lambda_{n}\left(\rho\left(t \wedge \tau^{s}, x\left(t \wedge \tau^{s}\right)\right)\right)\right]=\lambda_{n}(\rho(s, x)) \\
& \quad+E_{s, x}\left[\int_{s}^{t \wedge \tau^{s}}\left\{\frac{\lambda_{n}^{\prime \prime}(\rho)}{2} \sum_{\imath, j=1}^{d} a_{\imath \jmath} \rho_{x_{\imath}} \rho_{x,}+\lambda_{n}^{\prime}(\rho) H\right\}(u, x(u)) d u\right] \\
& =E_{s, x}\left[\int_{s}^{t \wedge \tau^{s}} \frac{\lambda_{n}^{\prime \prime}(\rho)}{2} \sum_{\imath, j=1}^{d} a_{\imath \jmath} \rho_{x_{i}} \rho_{x}(u, x(u)) d u\right] \\
& \quad+E_{s, x}\left[\int_{s}^{t \wedge \tau^{s}} \lambda_{n}^{\prime}(\rho) H(u, x(u)) d u\right]=I_{1}+I_{2}, \\
& I_{1} \leqq \\
& \quad \frac{1}{2} \int_{s}^{t} E_{s, x}\left[\frac{C_{3}}{n} \rho\left(u \wedge \tau^{s}, x\left(u \wedge \tau^{s}\right)\right) \rho^{-1}\left(u \wedge \tau^{s}, x\left(u \wedge \tau^{s}\right)\right)\right. \\
& \left.\quad \cdot \chi_{\left.1\left(u \wedge \tau s, x\left(u \wedge \tau^{s}\right)\right) \in W-\bar{D}\right)}\right] d u \leqq \frac{C_{3}}{n} \rightarrow 0 \quad(n \rightarrow \infty)
\end{aligned}
$$

and

$$
I_{2} \leqq C_{4} \int_{s}^{t} E_{s, x}\left[\rho\left(u \wedge \tau^{s}, x\left(u \wedge \tau^{s}\right)\right) \vee 0\right] d u
$$


where $\chi_{A}$ denotes the indicator function of the set $A$. Hence, letting $n \rightarrow \infty$, we obtain

$$
E_{s, x}\left[\rho\left(t \wedge \tau^{s}, x\left(t \wedge \tau^{s}\right)\right) \vee 0\right] \leqq C_{4} \int_{s}^{t} E_{s, x}\left[\rho\left(u \wedge \tau^{s}, x\left(u \wedge \tau^{s}\right)\right) \vee 0\right] d u
$$

for all $t \geqq s$ and each $(s, x) \in \partial D$. This implies $P_{s, x}\left[\left(t \wedge \tau^{s}, x\left(t \wedge \tau^{s}\right)\right) \in \bar{D}\right]=1$ and consequently $P_{s, x}[(t, x(t)) \in \bar{D}$ for all $t \geqq s]=1$ for each $(s, x) \in \partial D$. Then it follows from Corollary 4.1 that such a solution never leaves $\partial D$ and is unique. Hence our assertion is proved.

Q.E.D.

Remark 4.5. From the proof of Proposition 4.1, we have that any solution of the martingale problem $\left([0, \infty) \times R^{d}, a, b,(s, x) ; s \leqq t\right)$ starting in $\bar{D}$ satisfies (2.1), provided that the conditions [C.2.1], [C.2.2], [C.4.2] and the assumption [A.2.1] with $\phi \in \mathcal{H}^{3}\left([0, \infty) \times R^{d}\right)$ hold for $\partial D$. That is, $\bar{D}$ is an invarient set for any solution. This result is more general than Friedman's one [2]. It is, however, narrower than Theorem 2.1 in the sense of the existence of the solution on $\bar{D}$.

Remark 4.6. It is easily seen that a sufficient condition for [C.4.1] and [C.4.2] is that $a(t, x)$ and $b(t, x)$ are Lipschitz continuous with respect to the space variables $x$ on some neighborhood of $\partial D$ and $\phi(t, x)$ belongs to $\mathscr{H}^{3}\left([0, \infty) \times R^{d}\right)$. Hence, under this sufficient condition and assumptions in Proposition 4.1 except for [C.4.1] and [C.4.2], we get the result of Proposition 4.1. Especially, this case contains the case where $a(t, x)$ and $b(t, x)$ are Lipschitz continuous with respect to the space variables $x$ on $[0, \infty) \times R^{d}$ and $a(t, x)$ degenerates only on $\partial D$ so that at least [C.2.1], [C.2.3] and [A.4.2] hold. Moreover, in this case, if $d=2$, [A.4.2] is unnecessary because the uniqueness of the solution of the martingale problem on $\partial D$ is locally equivalent to the uniqueness of the solution of the onedimensional stochastic differential equation corresponding to the diffusion operator with the Lipschitz continuous coefficients (cf. [7]).

The uniqueness problem for the case where the path may enter the interior $D$ after hitting the boundary $\partial D$ is very difficult. Up to now, we have only obtained special examples of it, which appears in population genetics (see [4] and its references).

\section{REFERENCES}

[1] R.F. Anderson, Diffusions with second order boundary conditions, I, Indiana Univ. Math. J., 25 (1976), 367-395; II, 403-441.

[2] A. Friedman, Stochastic differential equations and applications, Vols. I-II, Academic Press, New York (1975 and 1976).

[3] N. OKaDA, On convergence to diffusion processes of Markov chains related to population genetics, Adv. Appl. Prob., 11 (1979), 673-700.

[4] N. OKADA, On the uniqueness problem of two dimensional diffusion processes 
occurring in population genetics, to appear.

[5 A. V. SKorohod, Studies in the theory of random processes, Kiev (1961). (English translation, Addison-Wesley, 1965)

[6- D.W. Strook AND S.R.S. VARAdHAN, Diffusion processes with continuous coefficients, I, Comm. Pure Appl. Math., 22 (1969), 345-400.

[7- T. YAmAda AND S. WATANABE, On the uniqueness of solutions of stochastic differential equations, J. Math. Kyoto Univ., 11 (1971), 155-167.

Department of Applied Phiysics

TOKyo Institute of TECHNOLOGY 